\title{
Absorption Spectra of Thioindigo Dyes in Benzene and Chloroform ${ }^{1}$
}

\author{
Wallace R. Brode and George M. Wyman
}

\begin{abstract}
The spectral absorption curves of 10 purified thioindigo dyes, containing mostly methyl and ethoxyl groups or halogens as substituents, in benzene and chloroform solution were determined over the ultraviolet and visible parts of the spectrum. The existence of an equilibrium between cis and trans isomers was indicated by the study of the effect of light on the absorption spectra. Some of the results of this investigation have been published. ${ }^{2}$ The present paper supplements the earlier paper and contains the spectral absorption curves that have not previously been published, along with the data on the purification of the three dyes that had not been reported there. In general, the first (long-wave) absorption band of the $c i s$-form of each dye occurs at a shorter (by approximately $60 \mathrm{~m} \mu$ ) wavelength than that of the corresponding trans isomer. The spectra are displaced slightly toward longer wavelengths in chloroform solutions than in benzene. Substituents in the five and seven positions cause a bathochromic shift in the spectra of both isomers of each dye in each solvent without affecting the shape of the spectral absorption curves. The introduction of substituents in the four or six positions results in a change in the shape of the absorption curves and a shift of the absorption to shorter wavelengths.
\end{abstract}

\section{Introduction}

The absorption spectra of the 10 purified thioindigo dyes listed in table 3 were determined as a part of the National Bureau of Standards research project on the spectra of pure dyestuffs. It was observed that the absorption spectra of these dyes in organic solvents were a function of the wavelength range of the illumination to which the solutions had been exposed prior to the measurement of the spectrum. This phototropic behavior was attributed to the existence of a dynamic equilibrium between the cis and trans forms (as shown here for thioindigo):

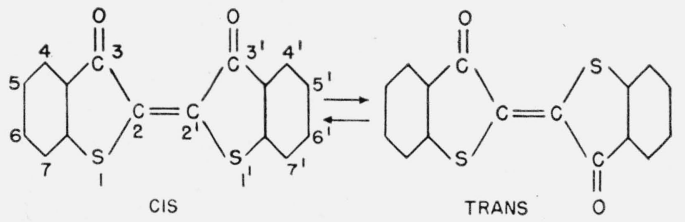

It was also possible to separate the two isomers in the case of two of these dyes. Some of the results of this investigation on seven of these dyes have been published (see footnote 2).

The object of this paper is to reproduce the complete spectral absorption curves of the dyes for which only the position of the absorption bands was reported in the earlier paper (cf. tables 2 and 3 of the reference cited in footnote 2); to describe the purification and the absorption spectra of three additional dyestuffs of this type; and to attempt to establish further correlations between the spectral absorption and the structure of these molecules.

Paper XXIII in the series on "The relation between the absorption spectra and the chemical constitution of dyes" (Paper X XII is referred to in footnote 2).

2 G. M. Wyman and W. R. Brode, J. Am. Chem. Soc. 73, 1487 (1951).

\section{Experimental Methods}

\subsection{Purification of Dyes}

The dyes used in this work were commercial products of known structure. Each was purified by adsorption on silica gel followed by selective elution. The solutions were then evaporated to dryness and the residue recrystallized from benzene or chloroform. Table 1 lists the conditions of the purification of the three dyes that had not been reported previously.

TABLE 1. Chromatographic purification of thioindigo dyes

\begin{tabular}{|c|c|c|c|}
\hline Dye & $\begin{array}{l}\text { Adsorbed } \\
\text { from- }\end{array}$ & Eluted with- & $\begin{array}{l}\text { Crystal- } \\
\text { lized from- }\end{array}$ \\
\hline $\begin{array}{l}5,5^{\prime}, 7,7^{\prime} \text {-Tetramethylthio- } \\
\text { indigo } \\
4,4^{\prime}, 5,5^{\prime}, 7,7^{\prime} \text {-Hexachloro- } \\
\text { thioindigo. } \\
\text { Vat Scarlet G- }\end{array}$ & $\begin{array}{l}\mathrm{CCl}_{4}{ }^{\mathrm{s}} \ldots \ldots \\
\mathrm{CCl}_{4} \\
\left\{50 \% \text { of } \mathrm{CCl}_{4}\right. \\
50 \% \text { of } \mathrm{C}_{6} \mathrm{H}_{6}\end{array}$ & 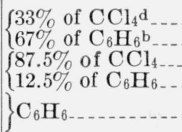 & $\left\{\begin{array}{l}\mathrm{CHCl}_{3} \mathrm{e} \\
\mathrm{CHCl}_{3} \\
\mathrm{CHCl}_{3}\end{array}\right.$ \\
\hline
\end{tabular}

Freshly distilled, technical.

b Freshly distilled, ACS grade.

c USP.

d By volume.

\subsection{Preparation of Solutions}

Approximately 0.01-g samples of each dye were weighed and dissolved in 600 to $700 \mathrm{ml}$ of benzene or chloroform by heating at the boiling point of the solvent for several hours. The solutions were then cooled to room temperature and diluted to 1 liter.

\subsection{Irradiation of Solutions}

The dye solution contained in a fused quartz absorption cell was exposed for 5 to $10 \mathrm{~min}$ to colored light, using a 100-w Spencer microfilm projector with 
Corning glass filters. These filters are listed in table 2, along with the manufacturer's numbers and the observed transmission cut-off (wavelength at which the transmission of light is less than $1 \%$ ).

\subsection{Measurement of Absorption Spectra}

The absorption spectra were measured at room temperature by means of a Cary Recording Quartz Spectrophotometer (Model 12), using matched absorption cells equipped with fused quartz windows.

\section{Results}

Figures 1 to 16 show the experimentally determined absorption spectra of eight of these thioindigo dyes in benzene and in chloroform solutions under the stated conditions of irradiation. Figures 17 to 32 present the calculated probable absorption curves for each pair of pure cis and trans isomers of each dye, which had not previously been reported, in the two solvents (concentration: $0.0100 \mathrm{~g} /$ liter, cell length: $2.00 \mathrm{~cm})^{3}$ Table 2 lists the approximate amounts of each isomer present at equilibrium, under various conditions of illumination, for the three dyes that had not previously been reported.

The spectral absorption curves in figures 1 to 16 demonstrate graphically the phototropic effect discussed in the previous paper (see footnote 2). This effect, attributed to the existence of a dynamic equilibrium between the cis and trans forms of these dyes, is apparently common to all thioindigo dyes

TABLE 2. Approximate amounts of the two isomers of each dye

\begin{tabular}{|c|c|c|c|c|c|}
\hline \multirow{2}{*}{ Corning filter number } & \multirow{2}{*}{$\begin{array}{l}\text { Wave- } \\
\text { length } \\
\text { region }\end{array}$} & \multicolumn{2}{|c|}{ In benzene } & \multicolumn{2}{|c|}{ In chloroform } \\
\hline & & trans & cis & trans & cis \\
\hline \multicolumn{6}{|c|}{$5,5^{\prime}, 7,7^{\prime}$-Tetramethylthioindigo $(565 \mathrm{~m} \mu) \mathrm{a}$} \\
\hline $\begin{array}{l}\text { None } \\
585 \\
351 \\
2424\end{array}$ & \begin{aligned} &$m \mu \\
&$\hdashline 495 \\
&$>520 \\
&>575\end{aligned}$ & $\begin{array}{l}\% \\
84 \\
89 \\
57 \\
29\end{array}$ & $\begin{array}{l}\% \\
16 \\
11 \\
43 \\
71\end{array}$ & $\begin{array}{l}\% \\
75 \\
78 \\
66 \\
44\end{array}$ & $\begin{array}{l}\% \\
25 \\
22 \\
34 \\
56\end{array}$ \\
\hline \multicolumn{6}{|c|}{$4,4^{\prime}, 5,5^{\prime}, 7,7^{\prime}$-Hexachlorothioindigo $(565 \mathrm{~m} \mu)^{\mathrm{a}}$} \\
\hline $\begin{array}{l}\text { None } \\
585 \\
351 \\
2424\end{array}$ & $\begin{array}{r}<495 \\
>520 \\
>575\end{array}$ & $\begin{array}{l}78 \\
82 \\
72 \\
45\end{array}$ & $\begin{array}{l}22 \\
18 \\
28 \\
55\end{array}$ & $\begin{array}{l}81 \\
84 \\
74 \\
55\end{array}$ & $\begin{array}{l}19 \\
16 \\
26 \\
45\end{array}$ \\
\hline \multicolumn{6}{|c|}{ Vat Scarlet G $\left(515 \mathrm{~m}_{\mu}\right)^{\mathrm{a}}$} \\
\hline $\begin{array}{l}\text { None } \\
585 \\
397 \\
351\end{array}$ & $\begin{array}{r}<495 \\
>350 \\
>520\end{array}$ & $\begin{array}{l}58 \\
68 \\
50 \\
28\end{array}$ & $\begin{array}{l}42 \\
32 \\
50 \\
72\end{array}$ & $\begin{array}{l}57 \\
63 \\
51 \\
29\end{array}$ & $\begin{array}{l}43 \\
37 \\
49 \\
71\end{array}$ \\
\hline
\end{tabular}

a Approximate wavelength of first absorption band.

${ }^{3}$ The experimentally observed absorption curves for thioindigo and $6,6^{\prime}$ diethoxythioindigo in the two solvents and the calculated probable absorption curves for these two dyes and for 4, $4^{\prime}$-dimethyl-6, $6^{\prime}$-dichlorothioindigo and bis-4,5benzothioindigo in chloroform were published in the previous paper (see footnote 2). in benzene and in chloroform solutions, and it was observed that even the hemi-thioindigoid dye, Vat Scarlet G (Colour Index No. 1228), exhibited this behavior. It is believed that interconversion of cis-trans isomers in general can be effected by selective irradiation, provided that the first absorption bands (at the long-wave end of the spectrum) of the two isomers occur at appreciably different wavelengths. It can be readily seen from figures 17 to 32 that the cis isomers of these dyes have their first absorption bands at a considerably higher frequency than do the corresponding trans forms. This frequency difference is remarkably constant and ranges from 1,800 to $2,250 \mathrm{~cm}^{-1}$ in chloroform; and from 2,000 to $2,400 \mathrm{~cm}^{-1}$ in benzene solutions for the 10 dyes reported here, as shown in table $3 .^{4}$

The absorption spectra of each dye in the two solvents used appear strikingly similar. There is a slight displacement of the first absorption bands toward lower wave numbers in chloroform. This displacement amounts to less than $200 \mathrm{~cm}^{-1}$ for the trans isomers and 200 to $400 \mathrm{~cm}^{-1}$ for the cis forms. It seems probable that the more polar solvent (chloroform) lowers the energy of the first excited states (which are also polar, cf. the reference cited in footnote 2), and this effect is more pronounced for the relatively more polar cis form than for the corresponding trans isomer. The ratios of the absorption bands are constant for each isomer and, for each dye, independent of the solvent (cf. table 3 ).

It is interesting to note that, although in the trans isomers, the most intense ultraviolet absorption peak is always at slightly less than double the frequency of the first absorption band (corresponding to the second harmonic of that fundamental vibration), this ratio is diminished considerably (to approximately 1.6 ) in the cis isomers (cf. table 3). Substitution in the $6,6^{\prime}$-positions reduces these ratios considerably and, in addition, gives rise to new absorption bands in the near ultraviolet.

It is possible to evaluate the effect of substituents by comparing the calculated absorption curves for the pure cis and trans isomers (figs. 17 to 32). It is immediately apparent from such a comparison that that the introduction of substituents in thioindigo in the 5 and 7 positions causes the usual shift toward longer wavelengths in both isomers. The effect of a methyl group is approximately equal to that of a chlorine atom (cf.figs. 17 to 23). This bathochromic shift is also observed when two bromine atoms are substituted in the 5, $5^{\prime}$-positions in $6,6^{\prime}$-diethoxythioindigo (cf. figs. 27 to 29). ${ }^{5}$ 4,4', 5,5', 7, $7^{\prime}$ Hexachlorothioindigo also shows this expected bathochromic shift in the ultraviolet region; the first absorption band in the visible region, however, is at about the same wavelength as that for tetramethylthioindigo. In its ultraviolet spectrum it shows the absence of the second, auxiliary absorption band in benzene solution only, thus contributing the sole

\footnotetext{
4 For an interpretation of this frequency difference of the reference in footnote 2 5 An explanation was suggested for these bathochromic shifts in the earlier paper (footnote 2).
} 
TABLE 3. Ratio of the frequency of each subsequent absorption band (in order of increasing wave numbers) to that of the first absorption band

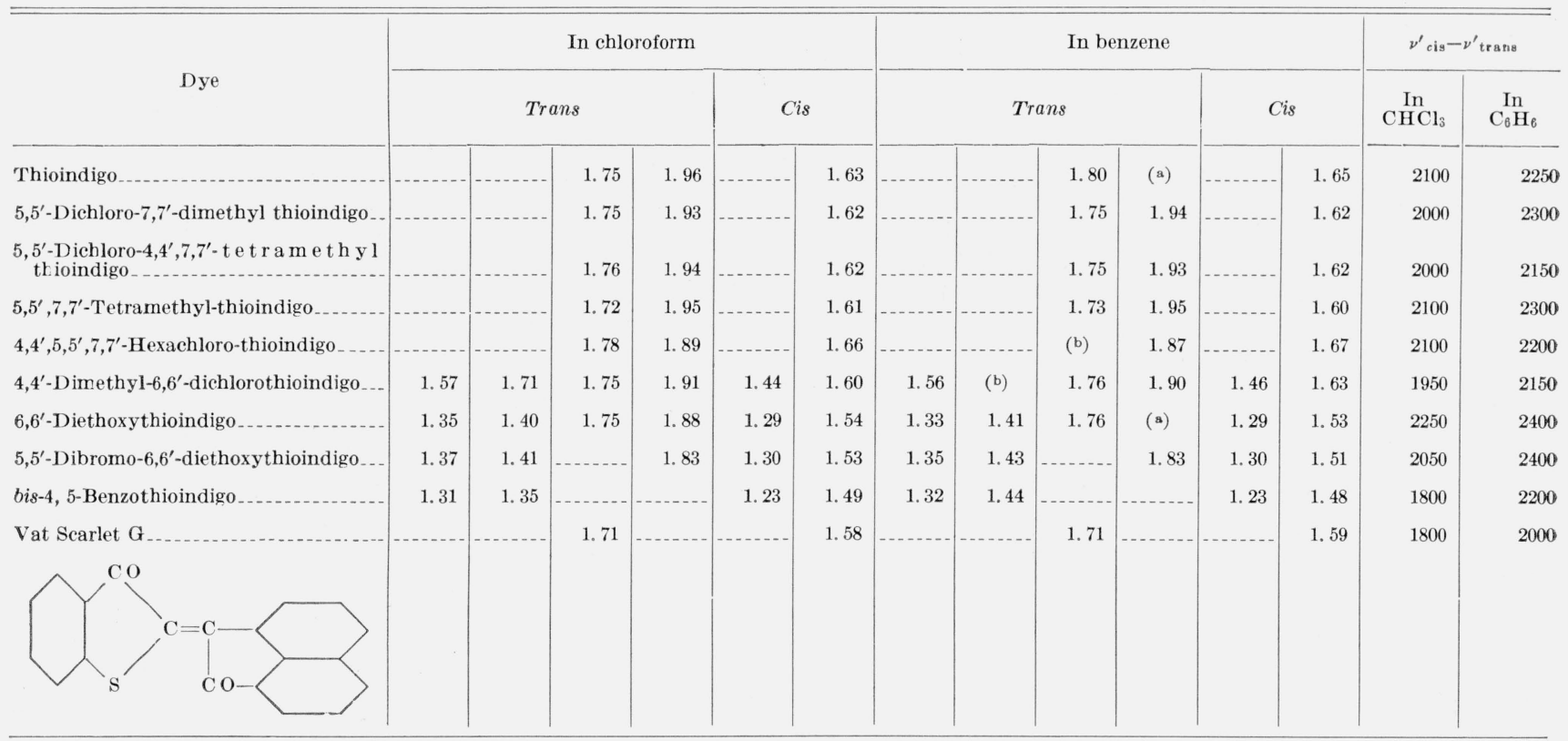

a Absorption band obscured by absorption due to solvent.

b A bsorption band absent in benzene, although present in chloroform.

example in this series for a compound showing a significant difference in absorption spectrum in the two solvents used (cf. figs. 7 and 8, also 24 and 25).

It had been pointed out by Formanek ${ }^{6}$ that the introduction of substituents in the $6,6^{\prime}$-positions in thioindigo causes a shift of the first absorption band toward higher frequencies. Although the existence of cis and trans isomers of thioindigo dyes was not known at that time, this statement has been shown to be applicable to the cis compounds also and an explanation for this, apparently anomalous, hypsochromic shift was suggested in the previous paper (see footnote 2). In general, the introduction of substituents in the $6,6^{\prime}$-position in the thioindigo nucleus gives rise to the appearance of new absorption peaks in the near ultraviolet region (cf. figs. 26 to 29 ). In addition, the most intense ultraviolet absorption band undergoes a slight shift toward lower frequencies in both isomers of each of these dyes (except trans 6,6'-diethoxy thioindigo). Thus, it appears likely that in the second excited state, the $6,6^{\prime}$-positions are more strongly conjugated with the negatively polarizable oxygen atoms, than they are in the first excited state. A comparison of the spectra of 5,5'-dichloro-7, $7^{\prime}$-dimethylthioindigo (figs. 17 and 18 ) and of $4,4^{\prime}$-dimethyl-6, $6^{\prime}$-dichlorothioindigo (figure 26 and figure 7 in the reference cited in footnote 2 shows, however, that the bathochromic shift of the main ultraviolet absorption band in the case of the latter is still far less than in the case of

${ }^{6}$ J. Formanek, Z. Angew. Chem. 41, 1137 (1928). its more "normal" isomer. It is interesting to note that the visible spectrum of bis-4,5-benzothioindigo (fig. 30 and fig. 9 in the reference cited in footnote 2 resembles the spectra of $6,6^{\prime}$-diethoxy thioindigo and of 5,5'-dibromo-6, $6^{\prime}$-diethoxy thioindigo more closely than that of any of the other thioindigo dyes. This suggests that the two phenyl groups act as electron donors similar to (though not as strong as) the ethoxy groups; contributing structures of this type to the resonance hybrid of the ground state:

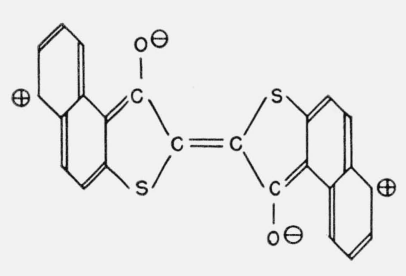<smiles></smiles>

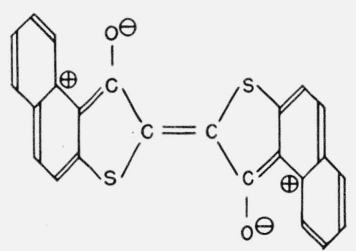

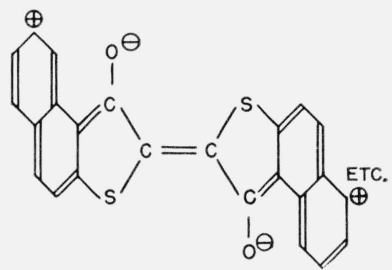

The strong ultraviolet absorption of bis-4,5-benzothioindigo (fig. 13) is probably due to the strong absorption normally associated with phenyl groups in this region of the spectrum. 


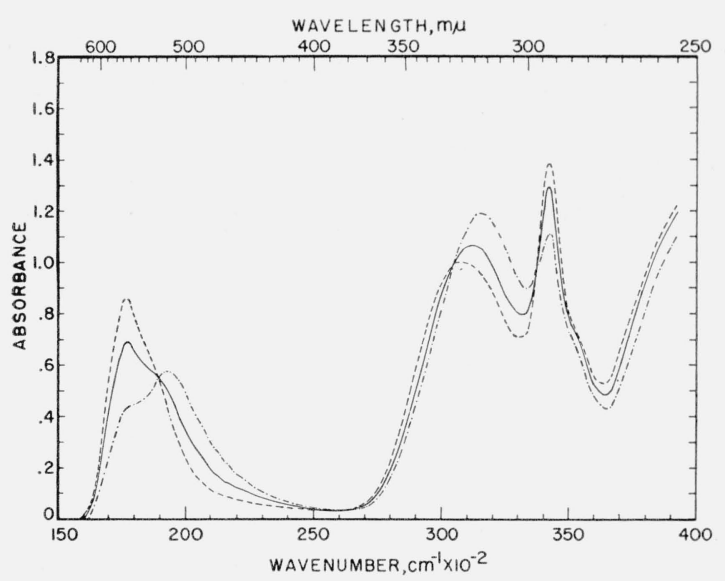

Figure 1. Absorption spectra of $5,5^{\prime}$-dichloro- $\gamma, \gamma^{\prime}$-dimethylthioindigo in chloroform.

light Exposed to blue light $(\lambda<495 \mathrm{~m} \mu)$; , exposed to yellow light $\overline{(\lambda>} \overline{520} \mathrm{~m} \mu) ;$
$0.0053 \mathrm{~g} /$ liter. Cell length,
$5.00 \mathrm{~cm}$.

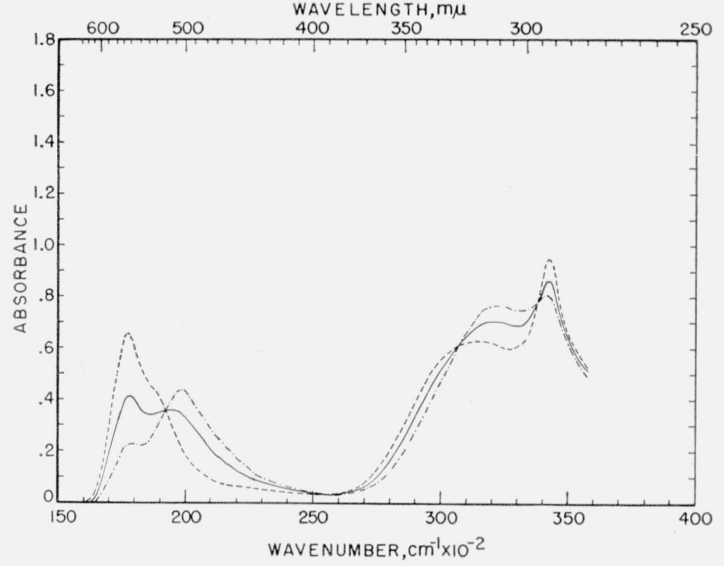

FiguRE 2. Absorption spectra of $5,5^{\prime}$-dichloro- $\gamma, \gamma^{\prime}$-dimethyl thioindigo in benzene.

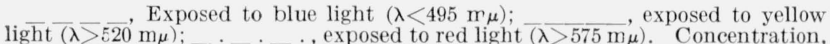
light $(\lambda>520$ m $\mathrm{m} \mu) ;$ $0.0091 \mathrm{~g}$ /liter. Cell ler $\overline{\mathrm{g}} \mathrm{th}, \overline{2} .60 \mathrm{~cm}$

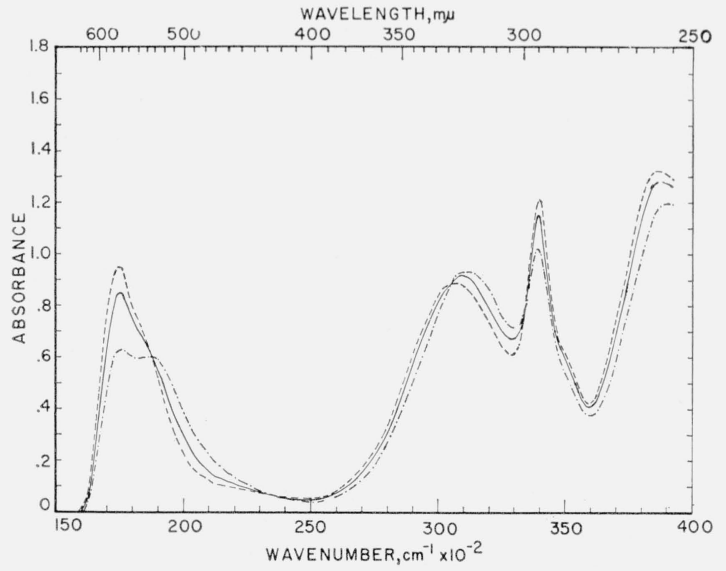

Figure 3. Absorption spectra of $5,5^{\prime}$-dichloro- $4,4^{\prime}, 7,7^{\prime}$-tetramethylthioindigo in chloroform.

, Exposed to blue light $(\lambda<495 \mathrm{~m} \mu)$;

, exposed to yellow light $\overline{(\lambda}>520 \mathrm{~m} \mu)$; $0.0117 \mathrm{~g} /$ liter. Cell length, $2.00 \mathrm{~cm}$.

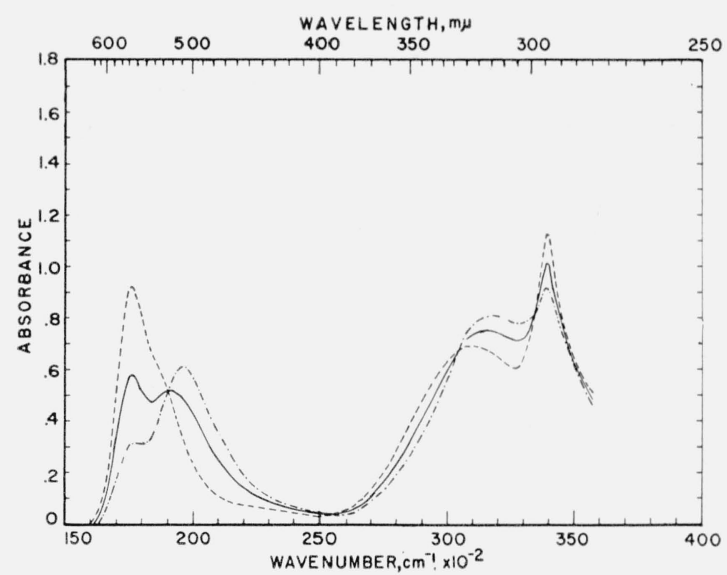

Figure 4. Absorption spectra of $5,5^{\prime}$-dichloro- $4,4^{\prime}, \gamma^{\prime}, 7^{\prime}$ tetramethylthioindigo in benzene.

light Exposed to blue light $(\lambda<495 \mathrm{~m} \mu)$; light $(\lambda>520 \mathrm{~m} \mu)$; . . . exposed to red light $(\lambda>575 \mathrm{~m} \mu)$. Concentration, $0.0108 \mathrm{~g} /$ liter. Cell length, $\overline{2} .00 \mathrm{~cm}$.

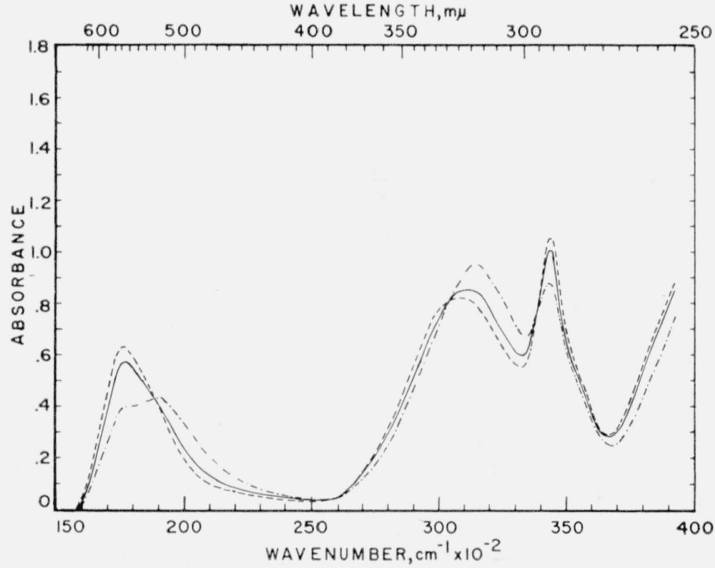

Figure 5. Absorption spectra of $5,5^{\prime}, 7,7^{\prime}$-tetramethylthioindigo in chloroform.

light $\ldots$, Exposed to blue light $(\lambda<495 \mathrm{~m} \mu)$; _ exposed to yellow light $\overline{(\lambda}>520 \mathrm{~m} \mu)$; $0.0091 \mathrm{~g} /$ liter. Cell length, $\overline{2} .00 \mathrm{~cm}$.

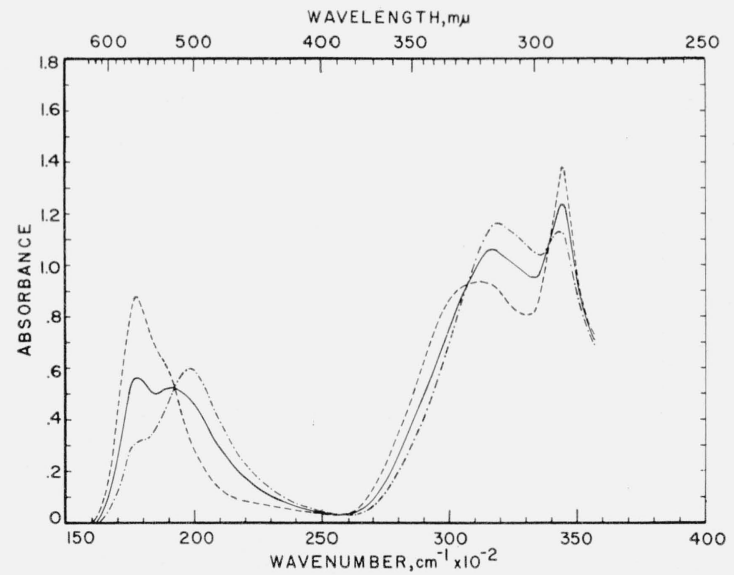

Figure 6. Absorption spectra of $5,5^{\prime}, \gamma, \gamma^{\prime}$-tetramethylthioindigo in benzene.

light Exposed to blue light $(\lambda<495 \mathrm{~m} \mu)$;

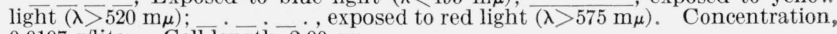
0.0107 g/liter. Cell length, $2.00 \mathrm{~cm}$ 


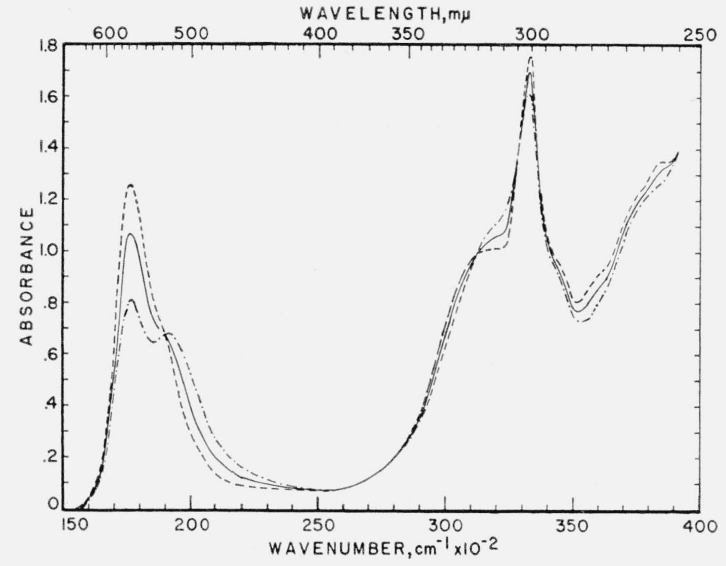

Figure 7. Absorption spectra of $4,4^{\prime}, 5,5^{\prime}, 7,7^{\prime}$-hexachlorothioindigo in chloroform.

Exposed to blue light $(\lambda<495 \mathrm{~m} \mu)$; , exposed to yellow light $\overline{(\lambda}>5 \overrightarrow{20} \mathrm{~m} \mu)$; . exposed to red light $(\lambda>575 \mathrm{~m} \mu)$. Concentration, $0.0055 \mathrm{~g} /$ liter. Cell length, $5.00 \mathrm{~cm}$.

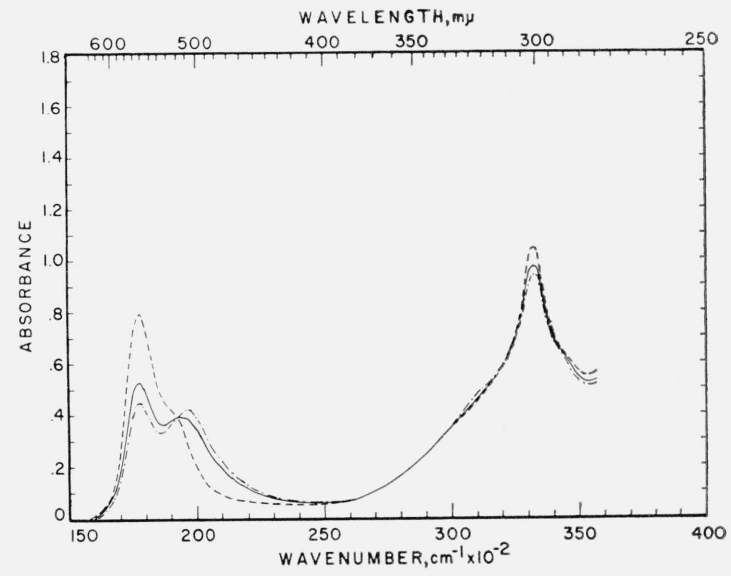

Figure 8. Absorption spectra of $4,4^{\prime}, 5,5^{\prime}, 7,7^{\prime}$-hexachlorothioindigo in benzene.

Exposed to blue light $(\lambda<495 \mathrm{~m} \mu)$, exposed to yellow light $\overline{(\lambda}>5 \overrightarrow{20} \mathrm{~m} \mu)$; $0.0096 \mathrm{~g} /$ liter. Cell length, $2.00 \mathrm{~cm}$.

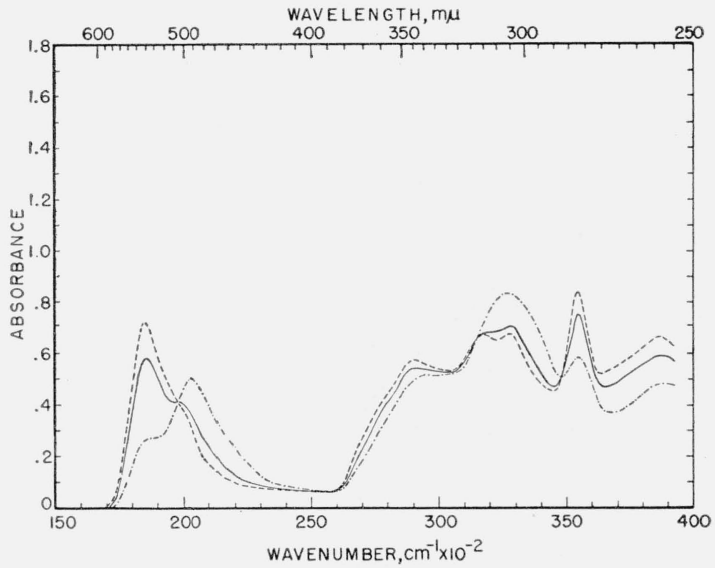

Figure 9. Absorption spectra of 4,4'-dimethyl-6,6'-dichlorothioindigo in chloroform. light $\overrightarrow{(\lambda}>350$ Exposed to blue light $(\lambda<495 \mathrm{~m} \mu) ; \underset{\text { m })}{\text {, exposed to green }}$ tion, $0.0081 \mathrm{~g} /$ liter. $-\dot{\text { Cell }}$ iength, $2.00 \mathrm{~cm}$.

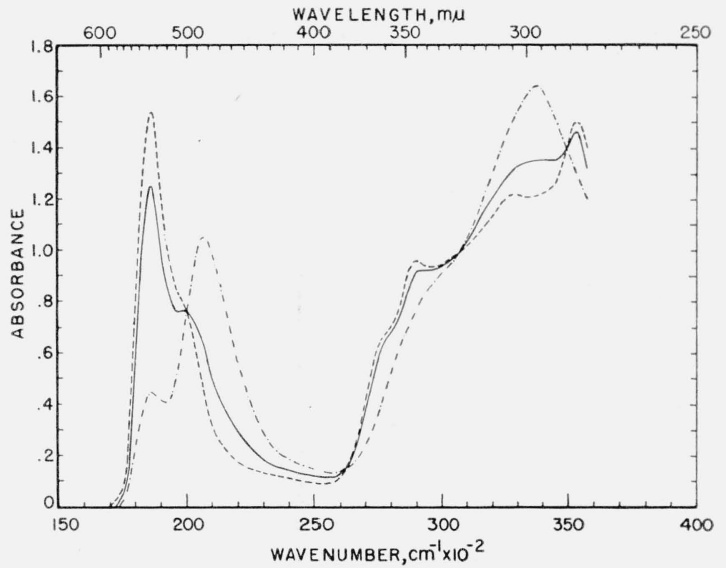

Figure 10. Absorption spectra of 4,4'dimethyl-6, $6^{\prime}$-dichlorothioindigo in benzene.

Exposed to blue light $(\lambda<495 \mathrm{~m} \mu) ;$ light $\overline{(\lambda}>350 \mathrm{~m} \mu) ;$. tion, $0.0144 \mathrm{~g} /$ liter. Cंell length, $2.00 \mathrm{~cm}$

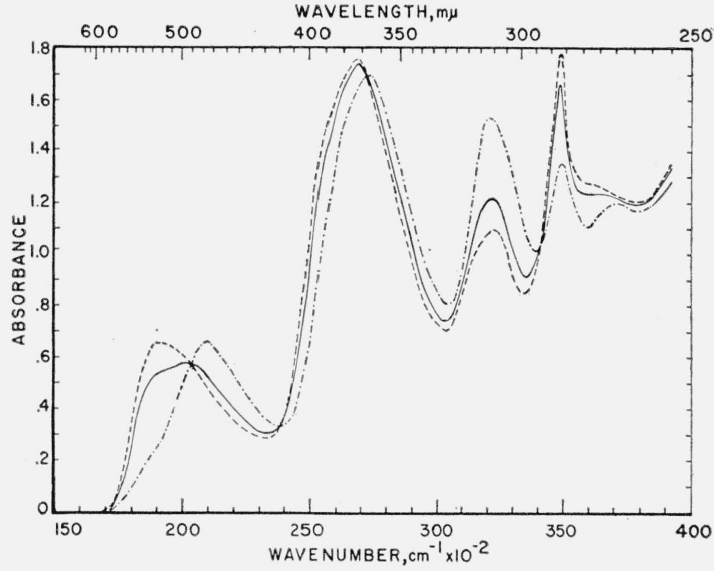

Figure 11. Absorption spectra of $5,5^{\prime}$-dibromo-6, $6^{\prime}$-diethoxythioindigo in chloroform.

Exposed to blue light $(\lambda<495 \mathrm{~m} \mu)$;

, exposed to daylight; exposed to yellow light $(\lambda>520 \mathrm{~m} \mu)$. Concentration, $0.0092 \mathrm{~g} /$ liter. Cell length, $5.00 \mathrm{~cm}$.

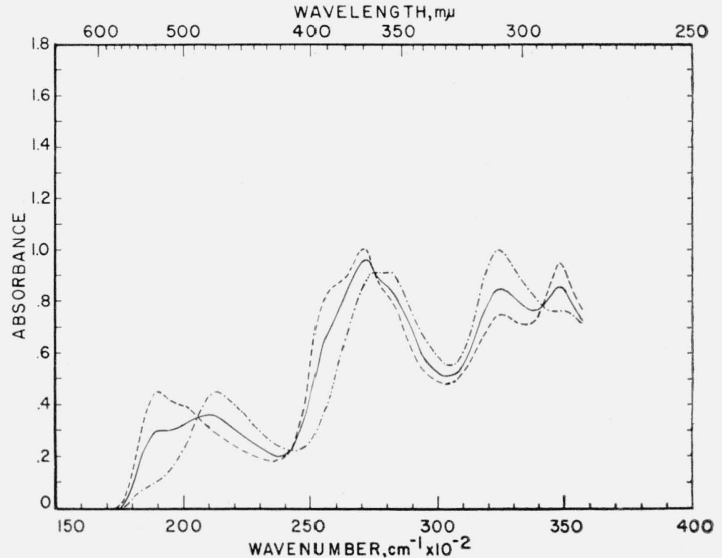

FIGURE 12. Absorption spectra of $5,5^{\prime}$-dibromo-6, $6^{\prime}$-diethoxythioindigo in benzene. light $\overline{(\lambda}>350$, Exposed to blue light $(\lambda<495 \mathrm{~m} \mu)$; light $\overline{(\lambda}>350$, Exposed to blue light $(\lambda<495 \mathrm{~m} \mu)$; tration, $0.0052 \mathrm{~g} /$ liter. Cell length, $5.00 \mathrm{~cm}$. 


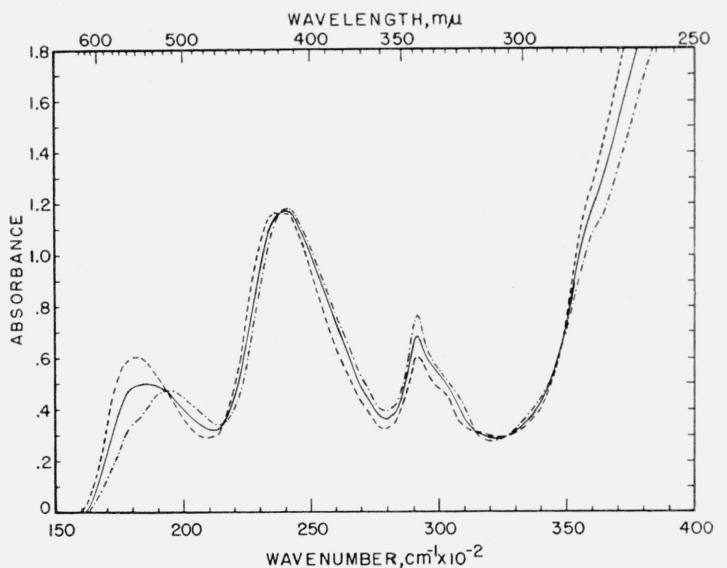

Figure 13. Absorption spectra of bis-4,5-benzothioindigo in chloroform.

Exposed to blue light $(\lambda<495 \mathrm{~m} \mu)$;

, exposed to yellow light $\overline{(\lambda}>4 \overline{70} \mathrm{~m} \mu)$; $0.0046 \mathrm{~g}$ /liter. Cell length, $\overline{5} .00 \mathrm{~cm}$.

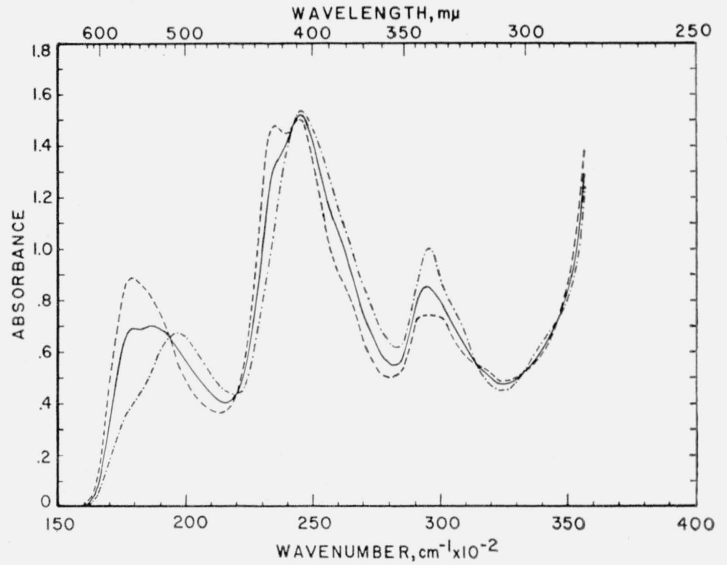

Figure 14. Absorption spectra of bis-4,5-benzothioindigo in benzene.

Exposed to blue light $(\lambda<495 \mathrm{~m} \mu)$;

exposed to yellow light $\overline{(\lambda}>470 \mathrm{~m} \mu) ;$ $0.0064 \mathrm{~g} /$ liter. Cél length, $\overline{5} .00 \mathrm{~cm}$.

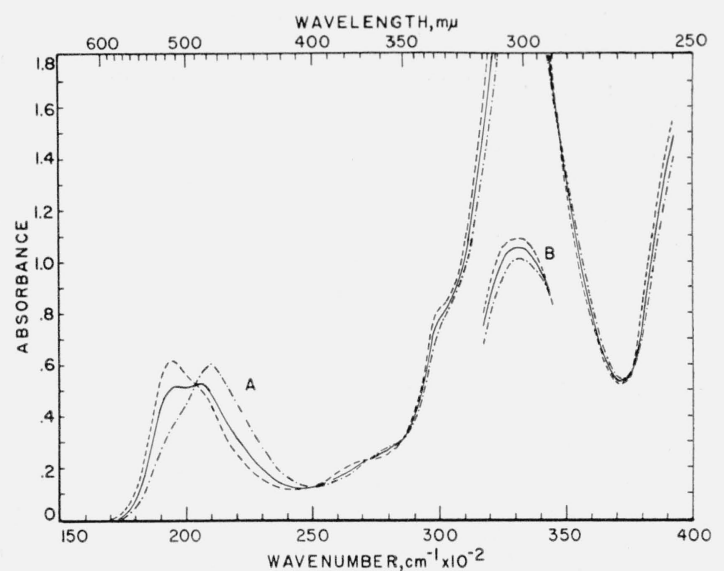

Figure 15. Absorption spectra of Vat Scarlet $G$ in chloroform. light $\longrightarrow$ Exposed to blue light $(\lambda<495 \mathrm{~m} \mu)$; light $(\lambda>350 \mathrm{~m} \mu)$;.$-{ }_{-}$. exposed to yellow light $(\lambda>520 \mathrm{~m} \mu)$. A, Concentration, $0.0109 \mathrm{~g} /$ liter. Cell length, $2.00 \mathrm{~cm}$. B, Concentration, $0.0055 \mathrm{~g} /$ liter.
Cell length, $2.00 \mathrm{~m}$.

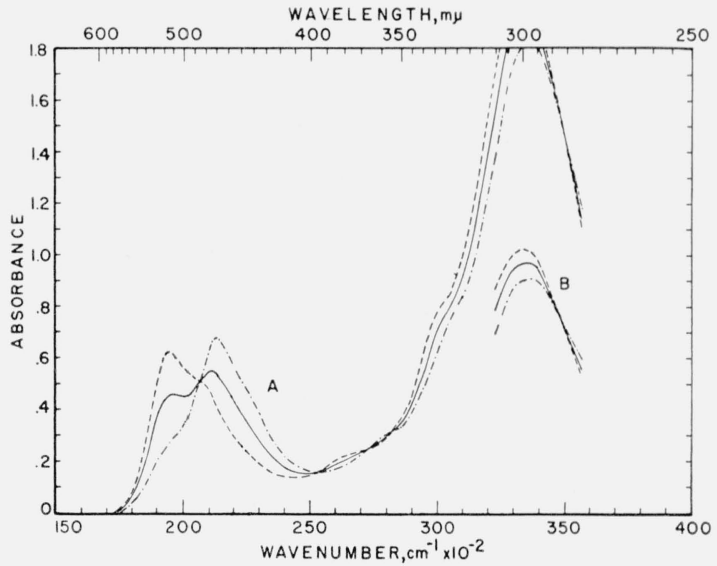

Figure 16. Absorption spectra of Vat Scarlet $G$ in benzene.

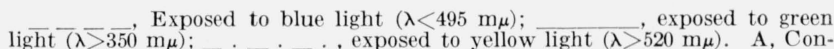
centration, $0.0100 \mathrm{~g} /$ liter. Cell length, $2.00 \mathrm{~cm}$. B, Concentration, $0.0050 \mathrm{~g} /$ liter. Cell length, $2.00 \mathrm{~cm}$

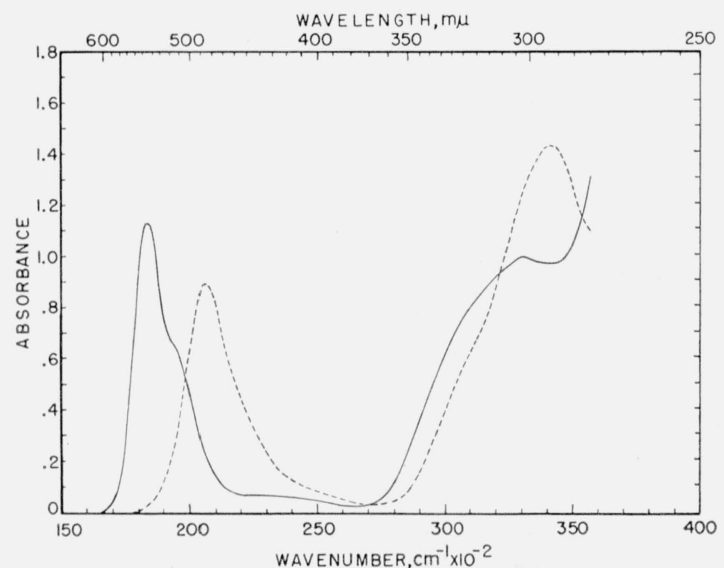

Figure 17. Calculated absorption spectra of thioindigo in benzene.

Trans-form; cis-form.

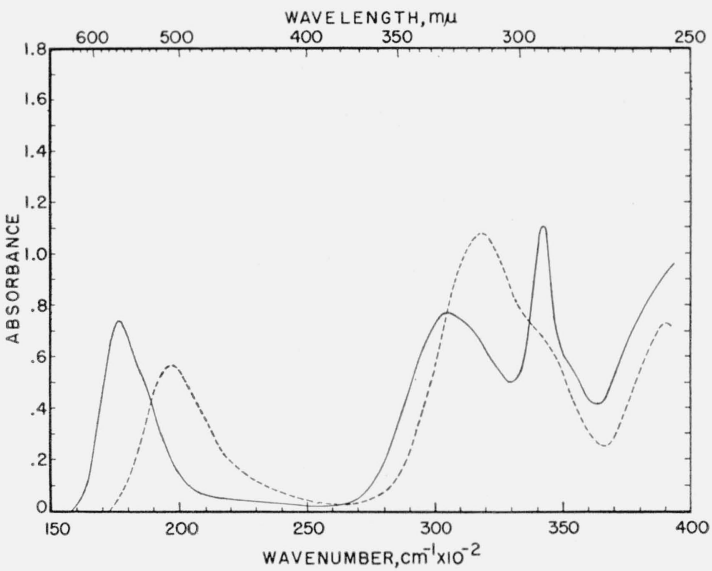

Figure 18. Calculated absorption spectra of 5,5'-dichloro- $7,7^{\prime}$ dimethylthioindigo in chloroform.

Trans-form; , cis-form. 


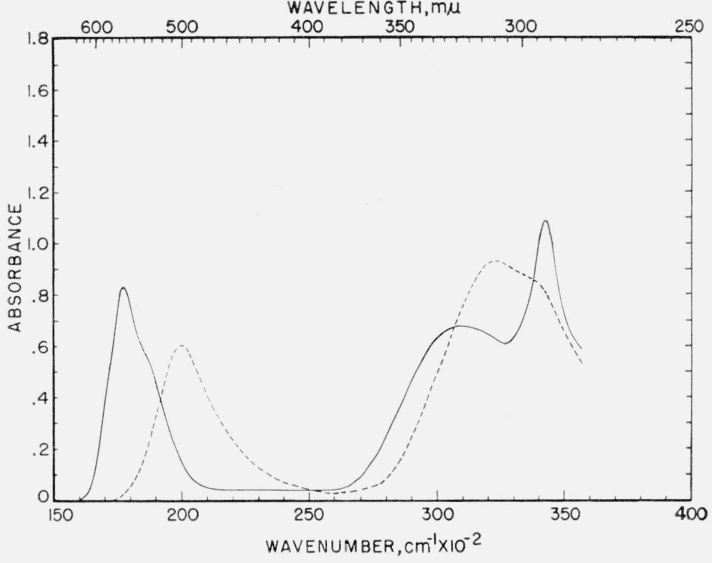

FIGURE 19. Calculated absorption spectra of 5,5'-dichloro$\gamma, \gamma^{\prime}$-dimethylthioindigo in benzene.

, Trans-form; , cis-form.

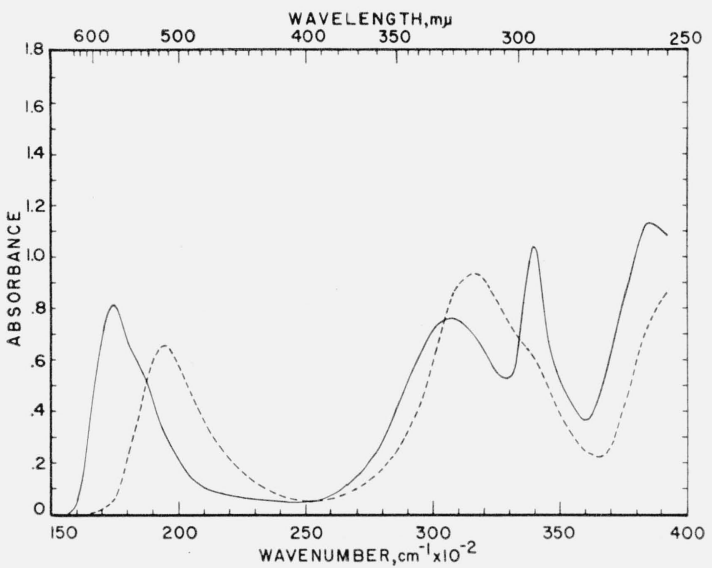

Figure 20. Calculated absorption spectra of 5,5'-dichloro-4, $4^{\prime}$, $\gamma, \gamma^{\prime}$-tetramethylthioindigo in chloroform.

, Trans-form , cis-form.

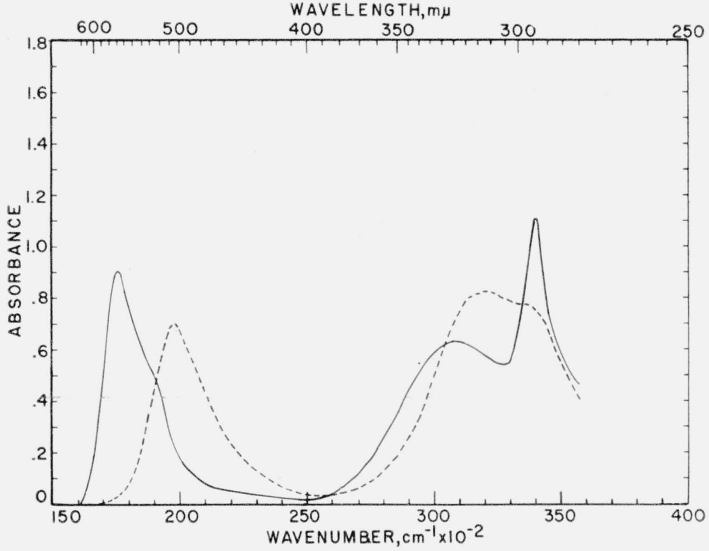

Figure 21. Calculated absorption spectra of 5,5'-dichloro-4,4', $7, \gamma^{\prime}$-tetramethylthioindigo in benzene.

Trans-form; cis-form.

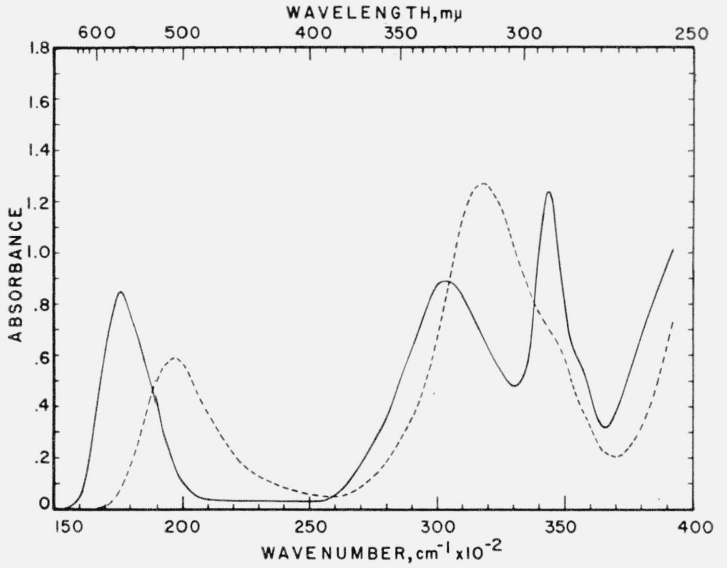

Figure 22. Calculated absorption spectra of 5,5', $7, \gamma^{\prime}$-tetramethylthioindigo in chloroform.

Trans-form; , cis-form.

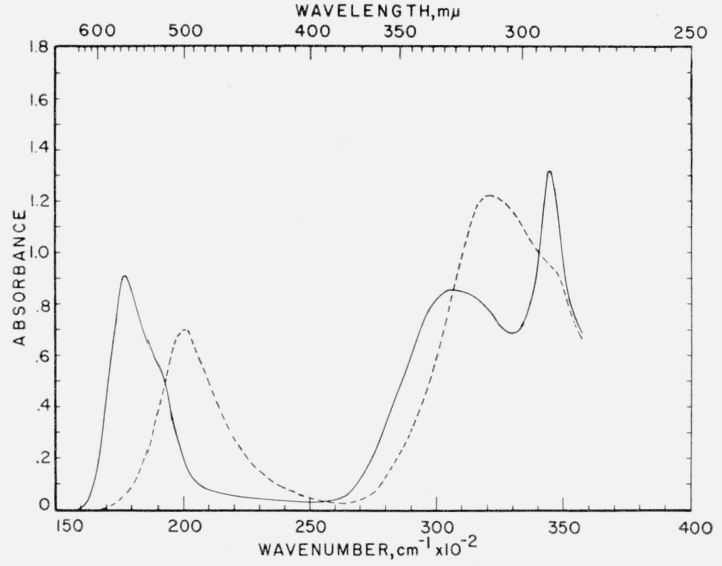

Figure 23. Calculated absorption spectra of 5,5', $7, \gamma^{\prime}$-tetramethylthioindigo in benzene.

Trans-form; , cis-form.

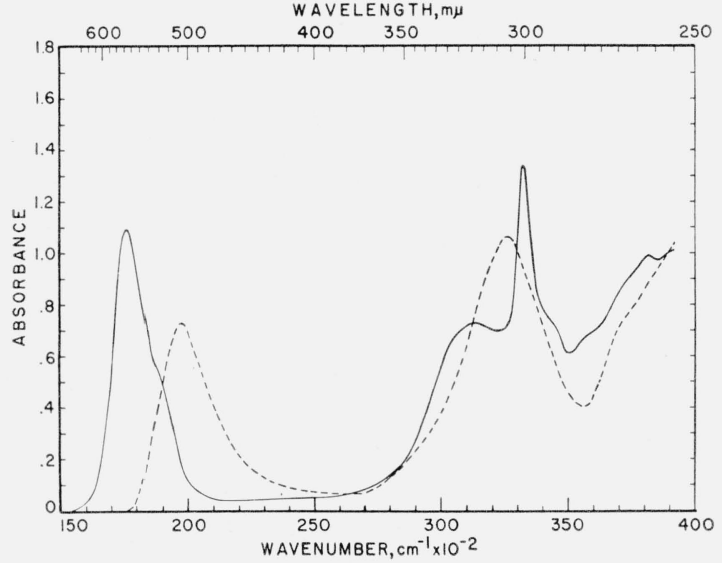

Figure 24. Calculated absorption spectra of $4,4^{\prime}, 5,5^{\prime}, 7,7^{\prime}-$ hexachlorothioindigo in chloroform.

Trans-form; , cis-form. 


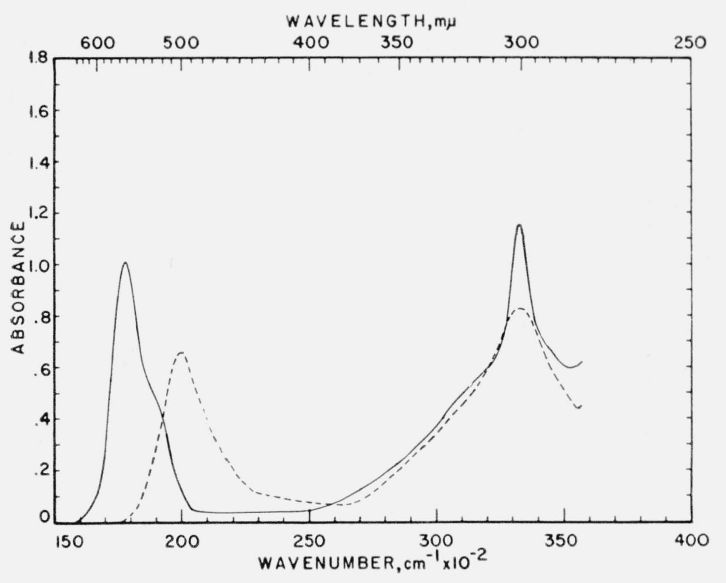

Figure 25. Calculated absorption spectra of $4,4^{\prime}, 5,5^{\prime}, 7,7^{\prime}$ hexachlorothioindigo in benzene.

, Trans-form; , cis-form.

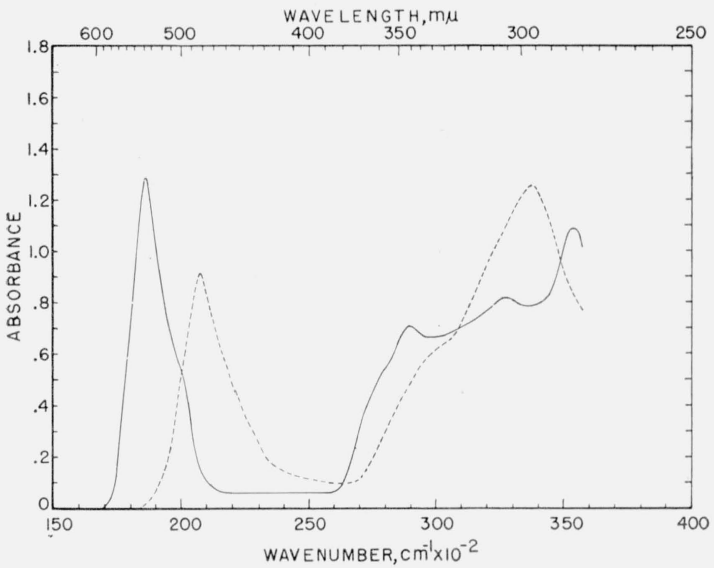

Figure 26. Calculated absorption spectra of 4,4'-dimethyl$6,6^{\prime}$-dichlorothioindigo in benzene.

Trans-form _, cis-form.

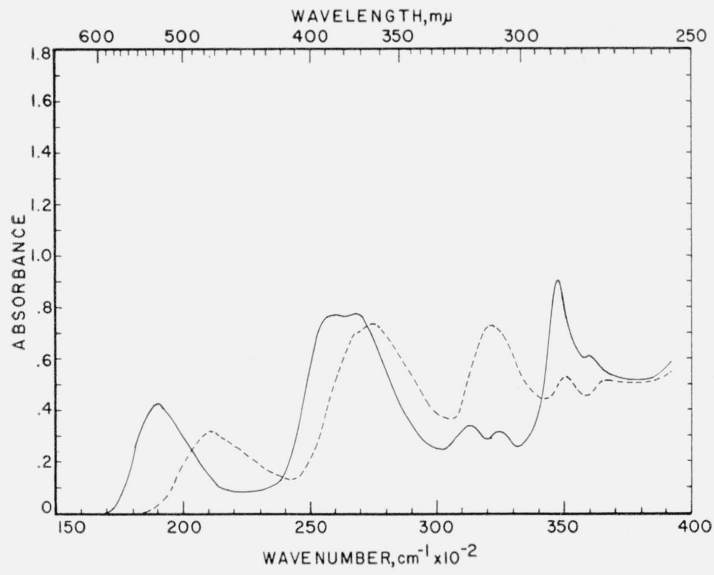

Figure 27. Calculated absorption spectra of 5,5'-dibromo-6,6'diethoxythioindigo in chloroform.

Trans-form; _ _ _ cis-form.

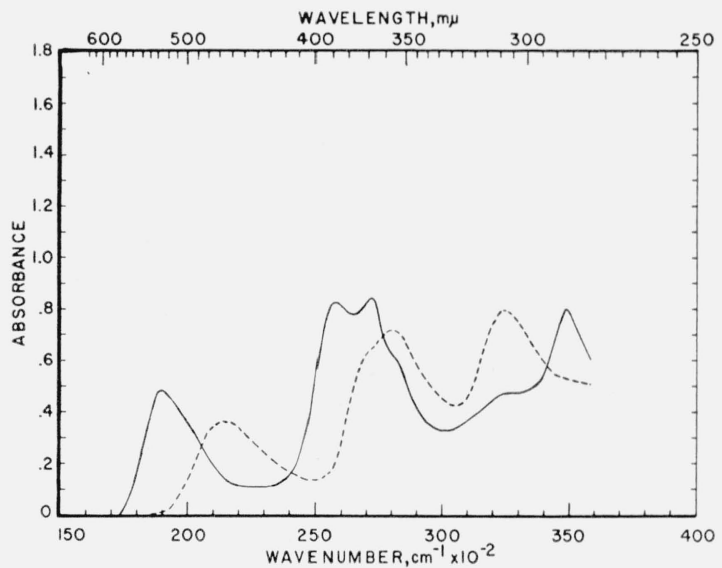

Figure 28. Calculated absorption spectra of 5,5'-dibromo$6,6^{\prime}$-diethoxythioindigo in benzene.

Trans-form; , cis-form.

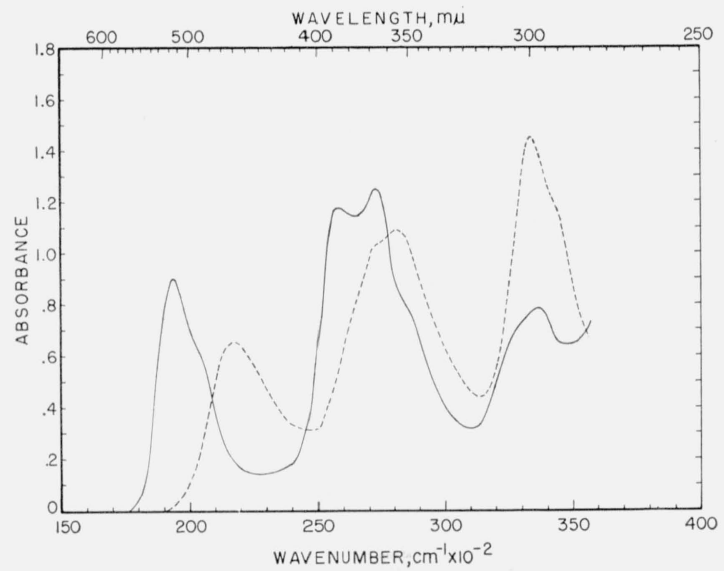

Figure 29. Calculated absorption spectra of 6,6'-diethoxythioindigo in benzene.

Trans-form; , cis-form.

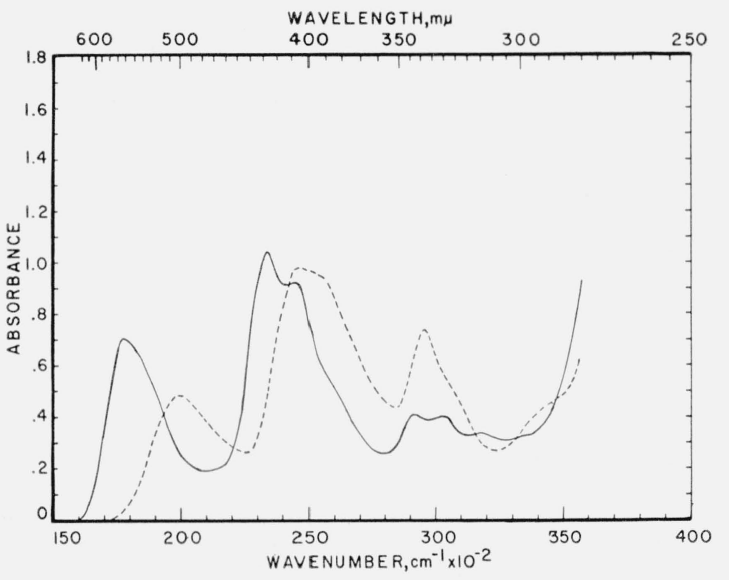

Figure 30. Calculated absorption spectra of bis-4,5-benzothioindigo in benzene.

Trans-form; cis-form. 


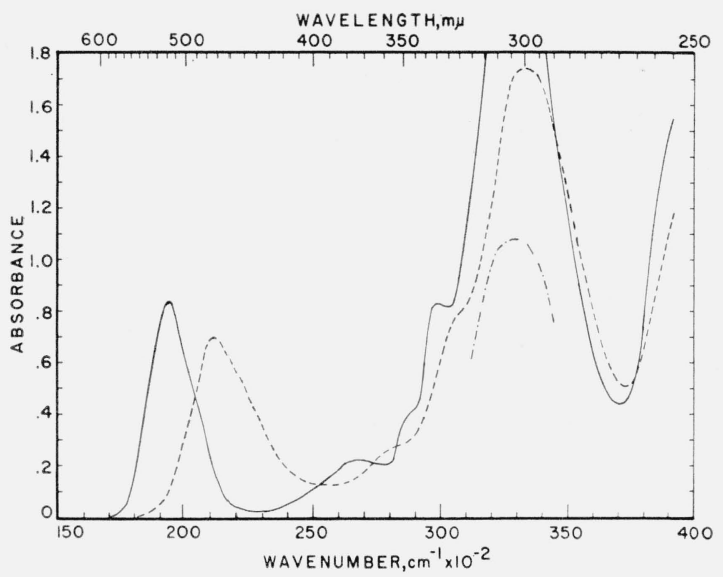

Figure 31. Calculated absorption spectra of Vat Scarlet $G$ in chloroform.

traction, $0.0050 \mathrm{~g}$ /liter.

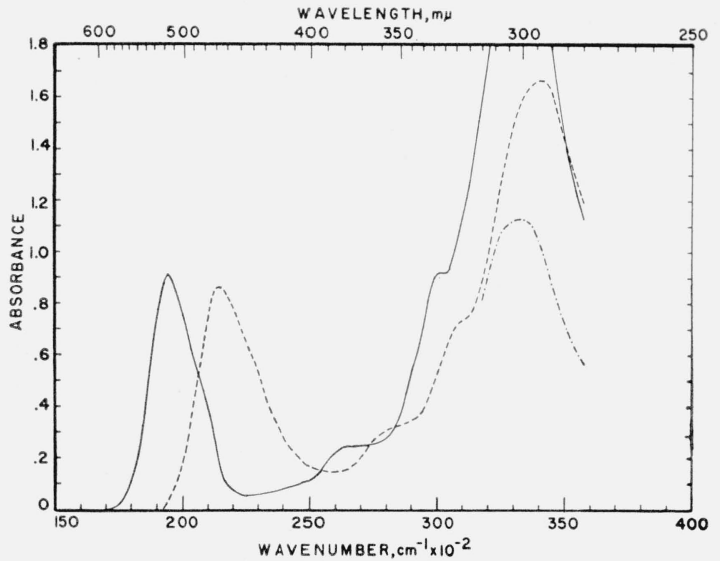

Figure 32. Calculated absorption spectra of Vat Scarlet $G$ in benzene.

Transform; _ _ _ _ cis-form; _ _ _ _ . , transform, concernration, 0.0050 g/liter.

Washington, March 5, 1951.

178 